\title{
Impact of crystal habits on interparticulate bonding strength of celecoxib
}

\author{
Dnyaneshwar P Kale ${ }^{1}$, Sameer R Modi ${ }^{1}$, Kailas S Khomane ${ }^{1}$, Arvind K Bansal ${ }^{1}$ \\ ${ }^{1}$ Pharmaceutics, National Institute Of Pharmaceutical Education And Research, Mohali, India \\ E-mail: dnyaneshniper@gmail.com
}

The present study investigates the influence of milling on differential compactibility behavior of two crystal habits of celecoxib (CEL) form III. Better compactibility of plate shaped (P-CEL) crystals over acicular (A-CEL) crystals was observed in unmilled samples, and compactability of both the habits were improved by milling. However, significantly different interparticulate bonding strength was observed in milled fractions of the two habits, in spite of similar particle size, shape, and surface area. The superior bonding strength of milled P-CEL (MP-CEL) over milled A-CEL (MA-CEL) was observed owning to the differential cleavage behavior of the two habits. Moreover, this differential cleavage behavior conferred the different surface molecular environment to the milled powders. On the surface of MP-CEL, the preferred cleavage of P-CEL across \{020\} plane exposed the -CF3 group and the methyl phenyl ring. Conversely, preferential breakage of A-CEL along their shortest axis resulted in the exposure of $\{100\}$ plane on the surface of MA-CEL, which uncovered the -CF3 group and the pyrazole ring. When studied by surface free energy quantification using advancing contact angle revealed greater dispersive component of MPCEL over MA-CEL. This is accordant with the differential cleavage behavior of P-CEL and A-CEL. This established the role of dispersive component of surface free energy in governing interparticulate bonding strength of CEL. The present study supports that the dispersive intermolecular interactions are more prominent factor over interparticulate bonding area in governing tablet tensile strength. In addition, this study also demonstrated the importance of selecting a suitable crystal habit of a drug to achieve the desired tensile strength of tablet dosage form, as the effect of crystal habit is pragmatically evident even after milling.

[1] Modi S. R. etal. (2014) Int J Pharm. 2, 460, 189-95

Keywords: Crystal habits, Bonding strength, Tableting 